\title{
FRI0106 RESULTS OF A SYSTEMATIC LITERATURE REVIEW OF PROGNOSTIC FACTORS IN RHEUMATOID ARTHRITIS AS A BASIS FOR A PROSPECTIVE RHEUMATOLOGISTS SURVEY
}

L. Carmona ${ }^{1}$, T. Otón ${ }^{1}$, A. Royo ${ }^{2}$, J.L. Baquero ${ }^{3}$, S. Luján ${ }^{2}$,

S. Muñoz-Fernández ${ }^{4}$. ${ }^{1}$ Instituto de Salud Musculoesquelética; ${ }^{2}$ Medical Dpt. Bristol-Myers Squibb; ${ }^{3}$ Departamento Científico, Scientia Salus; ${ }^{4}$ Rheumatology, Hospital Universitario Infanta Sofía, Universidad Europea, Madrid, Spain

Background: Selecting the most appropriate therapy for each patient with rheumatoid arthritis (RA) is crucial in order to prevent joint damage, particularly in patients with rapidly progressing disease. The literature on prognostic factors is tremendous, being practical to summarize which factors are most strongly associated with a particular outcome and what is the utility that rheumatologists assign to these factors.

Objectives: To identify well established factors predicting long-term outcomes in RA, as the basis for a survey.

Methods: The identification of the factors was performed via an overview of systematic reviews studying prognostic factors in RA, followed by scoping reviews for individual factors. All searches were conducted in PubMed. In order to be included in the overview, the study had to be a systematic review of prognostic factors of any of the following outcomes: disability, mortality, remission, response to treatment, or radiological damage. All factors identified, in positive or negative association with the selected outcomes, were compiled in a matrix of factors * outcomes. Subsequent scoping literature reviews were performed for each combination of the matrix.

Results: The overview of systematic reviews allowed the identification of 36 prognostic factors (see Table 1)

Table 1. Prognostic factors identified in the overview of systematic reviews

\section{Disability} Prognostic factors

Mortality Genetic markers
Quality of life index
Genetic markers
Medical history

No remission Medical history

Biomarkers

\begin{tabular}{|c|c|c|}
\hline & Biomarkers & $\mathrm{RF}$ \\
\hline & & ACPA \\
\hline & & RFA \\
\hline & & IL2 \\
\hline & & RANKL \\
\hline & & Vitamin D \\
\hline & Radiological data & Residual synovitis by US \\
\hline & & Scintigraphy \\
\hline & & MRI \\
\hline & Treatment & Treat to target \\
\hline & & Remission \\
\hline \multirow[t]{3}{*}{ No response to treatment } & Biomarkers & Calprotectin \\
\hline & & RF \\
\hline & Treatment & Treatment Failure \\
\hline \multirow[t]{9}{*}{ Structural damage } & Genetic markers & Shared epitope \\
\hline & & Polymorphisms \\
\hline & Radiological data & Erosions \\
\hline & Biomarkers & Calprotectin \\
\hline & & RF \\
\hline & & ACPA \\
\hline & & Composite activity indices \\
\hline & Treatment & Minimum treatment \\
\hline & & $\begin{array}{l}\text { Combination Therapy Effect } \\
\text { Recruitment time in clinical trials }\end{array}$ \\
\hline
\end{tabular}

After a round of discussions, we decided to remove all factors depending on treatment or study methodology for the scoping reviews, considering that they could not properly be considered prognostic factors but modifiers. Following the scoping reviews, we obtained a list of studies of prognostic factors, with methodological characteristics and 27 reviews of specific prognostic factors and outcomes. With this information, a survey addressed to practicing rheumatologists was developed to test how often they use the various factors to make long-term predictions, and how strong they think the association with outcome is.

Conclusions: We have analyzed and compiled a summary of prognostic factors published in RA and their predictability of long-term outcomes. This may act as a reference for cross-factor comparison and evidence-based risk assimilation and serve as a basis of surveying the value of such factors.

Acknowledgements: This study was funded by Bristol-Myers Squibb.

Disclosure of Interest: L. Carmona Grant/research support from: BMS, T. Otón Grant/research support from: BMS, A. Royo Employee of: BMS, J. L. Baquero Grant/research support from: BMS, S. Luján: None declared, S. Muñoz-Fernández Grant/research support from: BMS
DOI: 10.1136/annrheumdis-2017-eular.4765

\section{FRI0107 DEVELOPMENT OF A PREDICTIVE MODEL FOR RHEUMATOID ARTHRITIS MORTALITY USING RANDOM SURVIVAL FOREST}

L. Rodriguez-Rodriguez $^{1,2}{ }^{\text {, J.M. Lezcano }}{ }^{3}$, E. Soudah ${ }^{4}$, J.Á. Jover ${ }^{1}$,

I. González-Álvarez ${ }^{5,6}$, F. Salazar ${ }^{4}$, L. Abásolo ${ }^{1,2}$. ${ }^{1}$ Rheumatology, Hospital

Clinico San Carlos; ${ }^{2}$ San Carlos Health Research Institute, Madrid, Spain

${ }^{3}$ Rheumatology, San Carlos Health Research Institute; ${ }^{4} \mathrm{CIMNE}-M a d r i d ;{ }^{5} \mathrm{La}$

Princesa Health Research Institute; ${ }^{6}$ Rheumatology, Hospital de la Princesa,

Madrid, Spain

Background: Different machine learning methods have been used to develop predictive models of high quality and precision [1]. Among them, Random Survival Forests (RSF) has been proposed as an alternative to traditional survival models

[2], being able to overcome most of the limitation of traditional survival techniques, such as Cox proportional hazards models.

Objectives: Our objective was to develop and internally validate a predictive model for rheumatoid arthritis (RA) mortality using Random Survival Forests (RSF).

Methods: Retrospective longitudinal study involving 1,461 patients diagnosed with RA between January 1994 and August 2011, and followed at the outpatient clinic of the Rheumatology Department of the Hospital Clínico San Carlos (Madrid, Spain) until death or September 2013. Demographic and clinical-related variables collected during the first two years after disease diagnosis were used. RSF models were developed, based on 1,000 trees. 100 iterations of each model were performed to measure the mean and standard deviation (SD) of the predictive error and the integrated Brier score (IBS). Missing values were imputed using the function implemented by the randomForestSRC package [3]. The predictive capacity of the variables was assessed using the "variable importance" (VIMP). Two models were constructed using the log-rank $\left(M_{L G}\right)$ or log-rank score $\left(M_{L G S}\right)$ splitting rules. The model with the lowest prediction error was selected. Next, those variables with negative VIMP were excluded and a final model developed. Results: 148 patients died (10.1\%). $\mathrm{M}_{\mathrm{LG}}$ showed the lowest prediction error. All variables exhibited a positive VIMP. Final model showed a mean (SD) prediction error and IBS of $0.187(0.002)$ and $0.150(0.003)$ respectively. The most important predictor variables were age at diagnosis, median erythrocyte sedimentation rate and number of hospital admissions in the first 2 years after RA diagnosis.

Conclusions: We developed an accurate and precise model for RA mortality using RSF. Age and disease activity showed the highest influence in mortality.

References:

[1] Churpek MM, Yuen TC, Winslow C, Meltzer DO, Kattan MW, Edelson DP. Multicenter Comparison of Machine Learning Methods and Conventional Regression for Predicting Clinical Deterioration on the Wards. Crit Care Med. 2016;44: 368-74

[2] Ishwaran H, Kogalur UB, Blackstone EH, Lauer MS. Random survival forests. Ann Appl Stat. 2008;2: 841-860.

[3] Ishwaran H, Kogalur U. Random Forests for Survival, Regression and Classification (RF-SRC) [Internet]. 2016 [cited 15 Dec 2016]. Available: https://cran.r-project.org/package=randomForestSRC.

Disclosure of Interest: None declared

DOI: 10.1136/annrheumdis-2017-eular.6139

\section{FRI0108 TIME TO FIRST TREATMENT IS ASSOCIATED WITH A REFRACTORY COURSE OF RHEUMATOID ARTHRITIS}

M. Bécède ${ }^{1}$, F. Alasti ${ }^{1}$, L. Haupt ${ }^{2}$, A. Kerschbaumer ${ }^{1}$, U. Landesmann ${ }^{1}$, G. Supp ${ }^{1}$, J. Smolen ${ }^{1}$, D. Aletaha ${ }^{1} .{ }^{1}$ Department of Internal Medicine 3, Division of Rheumatology, Medical University of Vienna; ${ }^{2}$ Hietzing Hospital, Vienna, Austria

Background: It is an ongoing matter of research, whether the natural course of rheumatoid arthritis (RA) can be altered by an early intervention, a concept historically referred to as the "window of opportunity" (1). So far, only short-term disease activity outcomes have been investigated (e.g. "remission off drugs"), which are, however, inherently affected by the unknown rate of underlying rate self-limiting disease. It is unclear, whether among those, who eventually develop RA, the disease course is really affected by the timing of their initial treatment. Objectives: To explore whether the long-term course of RA is different according to the delay of initial treatment.

Methods: Based on a longitudinal observational dataset, we initially identified a group of patients with an observed refractory disease, we defined presenting with ongoing moderate or high disease activity (by the Simplified Disease Activity Index, SDAI), despite at least three courses of DMARDs, of which at least on course was a biological compound. To ensure that sufficient time had been allowed for the previous treatments to be exert their non-effects, we also required these patients to have total treatment time of at least 18 months in accordance with treat to target strategy ( $3 \times 6$ months).

We identified 399 patients with a treatment time of at least 18 months. 48 patients were excluded despite fulfilling the disease activity criteria, because they haven' experienced enough treatment courses, or had received a biological compound yet, to claim refractory disease as per our criteria above. We could include 69 refractory and 282 non-refractory patients in our analyses and then performed 
logistic regression analysis to assess the effects of different characteristics at baseline, including disease duration, on becoming refractory.

Results: By comparing patient characteristics (Table 1), more of the patients, who later will become refractory, are female $(94.2 \%$ Vs $73.4 \%, p>0.001)$, have higher baseline disease activity (SDAI of 25.5 vs $17.7, p<0.001$ ), and longer delay of the initial treatment from symptom onset ( $3.17 \mathrm{Vs} 1.34$ years, $p=0.001)$.

The multivariable logistic regression model confirmed that a longer delay of first treatment is independently afflicted with a higher probability of a refractory disease course at a later stage. This model was adjusted for disease activity at baseline and gender $(p<0.001$, Figure 1$)$. With increasing treatment delay, the chance of a dire disease course rises by approximately $1 \%$ every 6 months.

Table 1. Baseline characteristics in refractory and non-refractory patients

\begin{tabular}{|c|c|c|c|}
\hline Baseline Descriptive & $\operatorname{ReRA}(n=69)$ & non-ReRA $(n=282)$ & Sig. \\
\hline$\%$ Female & 94.2 & 73.4 & $<0.001$ \\
\hline$\% \mathrm{RF}+$ & 56.5 & 62.1 & 0.398 \\
\hline$\%$ ACPA + & 60.9 & 61.0 & 0.985 \\
\hline Time to Treatment* & $3.17(4.10)$ & $1.34(2.70)$ & 0.001 \\
\hline SDAI & $25.54(12.24)$ & $17.70(12.17)$ & $<0.001$ \\
\hline $\mathrm{CRP}^{\dagger}$ & $2.02(2.30)$ & $1.80(2.02)$ & 0.435 \\
\hline SJC 28 & $6.42(5.35)$ & $4.64(4.72)$ & 0.009 \\
\hline TJC 28 & $9.22(6.98)$ & $4.35(5.40)$ & $<0.001$ \\
\hline$E G A^{\ddagger}$ & 37.78 (20.49) & $28.77(21.40)$ & 0.002 \\
\hline $\mathrm{PGA}^{\ddagger}$ & $55.19(26.67)$ & $41.42(26.67)$ & $<0.001$ \\
\hline
\end{tabular}

${ }^{*}$ years; ${ }^{\dagger} \mathrm{mg} / \mathrm{dl} ;{ }^{\ddagger} 100 \mathrm{~mm}$ VAS scale.

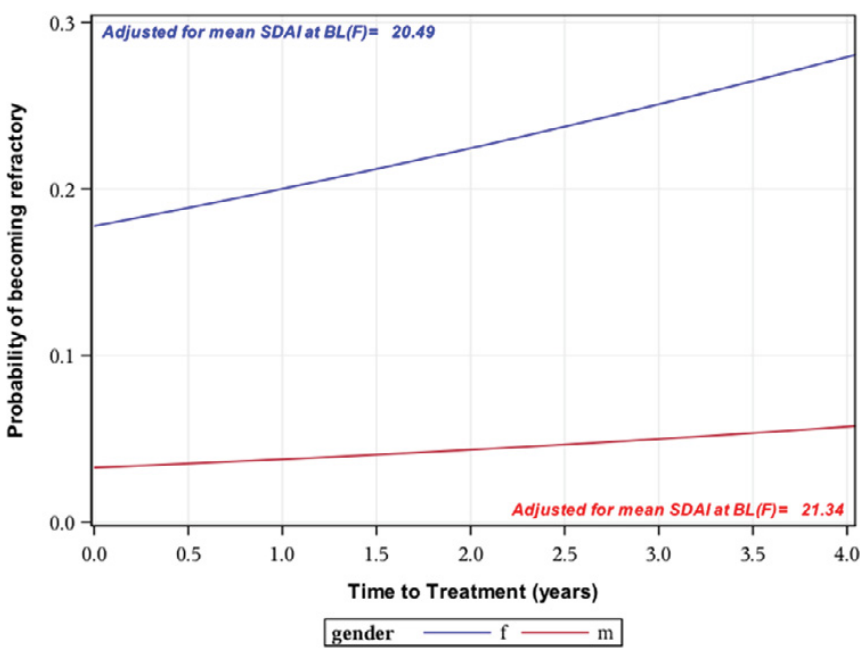

Figure 1: Predicted probabilities for becoming refractory depending on time to first treatment adjusted for baseline disease activity levels and gender.

Conclusions: Our data suggest that delay to initial treatment in RA affects the long-term course of RA. Earlier treatment initiation thus may change the severity of RA.

\section{References:}

[1] van Nies, J.A., et al., What is the evidence for the presence of a therapeutic window of opportunity in rheumatoid arthritis? A systematic literature review. Ann Rheum Dis, 2014. 73(5): p. 861-70.

Disclosure of Interest: None declared

DOI: 10.1136/annrheumdis-2017-eular.3946

\section{FRI0109 SHORT-TERM MONITORING OF SONOGRAPHIC CHANGES INDUCED BY A CORTICOSTEROID INJECTION IN METACARPOPHALANGEAL JOINT OF RHEUMATOID ARTHRITIS PATIENTS: A PILOT STUDY}

M. Di Carlo, D. Roia, A. Di Matteo, F. Salaffi, E. Filippucci, W. Grassi. Rheumatology Department, Università Politecnica delle Marche, Jesi, Italy

Background: Intra-articular corticosteroid injection is a well-established procedure in the daily practice since many decades [1]. High-resolution ultrasound (US), considered as in vivo microscopy, has the potential to help to understand how drugs develop their anti-inflammatory properties inside the articular space [2].

Objectives: To provide a very tight sonographic monitoring of the changes in a single metacarpophalangeal (MCP) joint in rheumatoid arthritis (RA) patients after the administration of intra-articular corticosteroid.

Methods: In this study we consecutively enrolled RA patients with active disease (Composite Disease Activity Index - CDAI >10), at least one tender and swollen MCP joint, and without contraindication to intra-articular corticosteroid injection (e.g. poor controlled diabetes). After the clinical evaluation that established the most clinically involved MCP joint to inject, patients underwent an US examination of the joint by an experienced sonographer. Synovitis was scored in grey scale (GS) in terms of joint space enlargement (measured at the level where the distance between the bone diaphysis and the joint capsule was greater), and power Doppler (PD) signal (scored by a semiquantitative method: $0=$ no intraarticular flow, 1 = single vessel signal, 2 = confluent vessels, and $3=$ vessel signal in more than $50 \%$ of the intra-articular area). After the baseline US assessment (T0), the MCP joints were injected with $20 \mathrm{mg}$ triamcinolone acetonide under sonographic guidance. Sonographic follow-up was performed in five timepoints: two hours (T1) after the injection, four hours (T2), eight hours (T3), 24 hours (T4), and 48 hours after the injection (T5).

Results: Fifteen patients ( 13 women), with a mean age of 62.5 years, completed the follow-up. The mean CDAI was 28.9, anti-citrullinated protein antibodies were present in 9 patients. At T5, in all the patients was detectable a global reduction of joint space enlargement, of intra-articular PD signal, and of the numerical rating scale (NRS) of pain at the joint injected (Table). However, in majority of the patients ( $n: 13$ ), the joint space enlargement showed an increase in the T1 and T2 US examinations, and in four patients PD signal, compared to T0, increased within the eight hours after the injection. No major adverse events were registered.

\begin{tabular}{|c|c|c|c|c|c|c|c|c|c|}
\hline \multirow[b]{2}{*}{ Case ID } & \multicolumn{3}{|c|}{$\begin{array}{c}\text { Power Doppler grade } \\
(0-3)\end{array}$} & \multicolumn{3}{|c|}{$\begin{array}{l}\text { Joint space enlargement } \\
(\mathrm{mm})\end{array}$} & \multicolumn{3}{|c|}{$\begin{array}{l}\text { NRS pain } \\
(0-10)\end{array}$} \\
\hline & First & Last & Maximum & First & Last & Maximum & First & Last & Maximum \\
\hline 1 & 2 & 1 & 3 & 3,4 & 2,4 & 4,2 & 3 & 1 & 5 \\
\hline 2 & 2 & 0 & 2 & 4,4 & 3,7 & 5,7 & 8 & 1 & 8 \\
\hline 3 & 2 & 0 & 2 & 2,1 & 1,3 & 3 & 7 & 2 & 7 \\
\hline 4 & 3 & 1 & 3 & 2,1 & 1,8 & 2,9 & 9 & 0 & 9 \\
\hline 5 & 3 & 2 & 3 & 4 & 4 & 4,9 & 10 & 0 & 10 \\
\hline 6 & 3 & 1 & 3 & 6,5 & 2,7 & 6,5 & 2 & 1 & 2 \\
\hline 7 & 2 & 0 & 2 & 3,5 & 1,2 & 3,6 & 6 & 0 & 6 \\
\hline 8 & 1 & 1 & 3 & 4,6 & 3,3 & 4,6 & 3 & 0 & 3 \\
\hline 9 & 3 & 0 & 3 & 2,5 & 2,1 & 3,6 & 2 & 0 & 2 \\
\hline 10 & 2 & 1 & 3 & 4,3 & 3,9 & 5,6 & 7 & 3 & 7 \\
\hline 11 & 3 & 1 & 3 & 5,3 & 3,3 & 5,7 & 8 & 3 & 8 \\
\hline 12 & 3 & 1 & 3 & 3,3 & 2,6 & 3,3 & 7 & 3 & 7 \\
\hline 13 & 2 & 1 & 2 & 2,6 & 2,3 & 3 & 7 & 2 & 7 \\
\hline 14 & 3 & 1 & 3 & 3,1 & 2,2 & 4 & 7 & 2 & 7 \\
\hline 15 & 2 & 1 & 3 & 4,5 & 3,7 & 4,5 & 7 & 0 & 7 \\
\hline
\end{tabular}

Conclusions: A single intra-articular corticosteroid injection, performed under US guidance, is a very fast treatment to reduce synovitis of the injected joint. In the hours next to the injection is common to reveal a rise of joint space enlargement together with that of PD signal score.

References:

[1] Courtney $P$, Doherty $M$. Joint aspiration and injection and synovial fluid analysis. Best Pract Res Clin Rheumatol 2013;27:137-69.

[2] Filippucci E, lagnocco A, Salaffi F, et al. Power Doppler sonography monitoring of synovial perfusion at the wrist joints in patients with rheumatoid arthritis starting adalimumab treatment. Ann Rheum Dis 2006; 65:1433-7.

Disclosure of Interest: None declared

DOI: 10.1136/annrheumdis-2017-eular.5357

\section{FRI0110 METHOTREXATE MONOTHERAPY IN REAL LIFE: A DRUG SURVIVAL ANALYSIS. COMPARISON BETWEEN VERY EARLY ARTHRITIS AND EARLY ARTHRITIS COHORTS}

M.G. Anelli ${ }^{1}$, C. Rotondo ${ }^{2}$, G. Righetti ${ }^{2}$, A. Rinaldi ${ }^{2}$, S. Perniola ${ }^{2}$, M. Nivuori ${ }^{2}$, S. Lopriore ${ }^{2}$, G. Lopalco ${ }^{2}$, G. Laselva ${ }^{2}$, C. Scioscia ${ }^{1}$, F. Cacciapaglia ${ }^{2}$ G. Lapadula ${ }^{2}, \mathrm{~F}$. lannone $2 .{ }^{1}$ DIM; ${ }^{2}$ DETO, Unit of Rheumatology, Bari, Italy

Background: Methotrexate (MTX) is the first line drug suggested in the ACR/EULAR guidelines to treat the rheumatoid arthritis (RA) (1) and spondylarthritis (SpA). Although MTX efficacy is demonstrated by high levels of evidences, maximum benefit might require interventions even earlier. For this reason, it is suggested to identify the patients (pts) with symptom onset of less than 12 weeks, that is very early arthritis, to obtain better outcomes. Few data are available by the clinical practice on MTX monotherapy (MTXm) survival in very early or early arthritis pts.

Objectives: We aim to evaluate the presence of different outcomes in MTXm drug survival and MTX efficacy between Very Early arthritis (VEA) pts (less than 12 weeks from symptom onset) and Early arthritis (EA) pts (12-52 weeks from symptom onset), and between early RA pts and early SpA pts.

Methods: On 305 pts, we selected 219 pts (30 pts diagnosed as VEA (4,2 (4) weeks from symptom onset) and 155 pts as EA (25,7 (25) wks from symptom onset) in which MTXm could be started. The RA pts were 93 and SpA pts were 126. To assess the MTXm persistency the Kaplan-Meier survival curves analysis was performed and Cox regression analysis was used to assess clinical predictors of MTXm suspension. The results are expressed as mean \pm standard deviation or median (interquartile range). A $p \leq 0.05$ was set for statistically significant.

Results: No difference in MTXm survival was found between VEA pts 95,5 (36) wks and EA 92,5 (10) wks ( $p=0,38)$ (Fig.1a). We observed a significant difference in drug survival between early RA pts 63 (16) wks) and early SpA pts 112,7 $(15,8)$ wks ( $p=0,001)$ (Fig. 1b). In $55 \%$ early RA pts and in $45 \%$ early SpA pts the biotechnology drug needs to be added because of disease flare. In the remaining pts a low disease activity (DAS $<3,2)$ was observed. No adverse events were recorded in follow-up period. In early RA pts predictors of shorter MTXm survival were younger age at diagnosis $(p=0,004)$, lower dose of MTX $(p=0,032)$, 